\title{
INCORPORATION OF UNCERTAINTY IN INVERSE PROBLEMS *
}

\author{
H.T. BANKS \\ Center for Research in Scientific Computation \\ North Carolina State University \\ Raleigh, $N C 27695$ \\ e-mail:htbanks@eos.ncsu.edu
}

\begin{abstract}
Motivated by examples from biology, electromagnetics and composite materials, we formulate a generic inverse problem for estimation of parameters represented as random variables. A general theoretical framework is outlined and applied to a model for the HIV infection pathway.
\end{abstract}

\section{Introduction}

In this lecture we present a summary of some of our experiences in the Industrial Applied Mathematics Program (IAMP-see www.ncsu.edu/crsc/iamp.html) at North Carolina State University. This program involves a number of projects (23 projects in 2000-2001) with industrial and nonacademic lab research groups. These projects incorporate certain common elements, especially with regard to sources of uncertainty.

In particular, we find that uncertainty is important in several distinct aspects including: $i$.data acquisition and related (sensor) observation error; ii.modeling intra-and inter-individual variability in systems and data. These aspects arise in diverse applications including inverse problems involving composite materials, biological systems, and electromagnetic imaging.

There are a number of approaches and tools critical to these efforts. These include:

$i$. Sensitivity analysis (with respect to parameters, geometry, etc.);

ii. "Dispersion" - type modeling and random parameters (to treat intraindividual variability);

iii. Random (stochastic) parameters and mechanisms (to treat interindividual variability in aggregate data and observations) in the context of "mixed effects" / "mixing distributions" modeling; and

*INVITED LECTURE, INTERNATIONAL CONFERENCE ON INVERSE PROBLEMS, CITY UNIVERSITY OF HONG KONG, JANUARY 9-12, 2002

ws-p8-50x6-00HTB: submitted to World Scientific on March 16, 2002 
$i v$. Model reduction techniques including reduced order dynamic models for complex systems (e.g., Proper Orthogonal Decomposition on damages see Banks et $\mathrm{al}^{10}$, etc.).

A central feature of each effort is an inverse or parameter estimation problem which can be succinctly stated as follows.

Generic Inverse Problem: One is given a set of data $\hat{d}=\left\{d_{i}\right\}$ corresponding to (perhaps partial) observations, $C y\left(t_{i} ; \pi\right)$, of the state $y$. The state dynamics are given by a parameter $(\pi)$ dependent system

$$
\frac{d y}{d t}=f(t, y(t), \pi)
$$

where $f$ can represent ordinary, functional, or partial differential equations and where $\pi$ is a (possibly vector valued) random variable. In a least squares setting, the problem is to minimize

$$
J(\pi, \hat{d})=\sum_{i}\left|C y\left(t_{i} ; \pi\right)-d_{i}\right|^{2}
$$

over $\pi \in \Pi=$ subject to (1), where $\Pi$ is a given family of random variables and $C$ is an observation operator.

This generic problem, of course, includes as special cases the usual problems with constant R.V.'s (i.e., the usual vector or function space parameters that are not dependent on any measure or variable of uncertainty).

In the next section we mention briefly several problems that can be formulated in this generic framework.

\section{Examples}

\subsection{PBPK Models for TCE in Fat Cells}

In Albanese et $\mathrm{al}^{1}$, Banks and Potter ${ }^{13}$, Banks and Potter ${ }^{14}$, Potter ${ }^{22}$, the authors consider models for the distribution of trichloroethylene (a cleaning solvent) in adipose tissue. The site of interest (the fat tissue "compartment" in the body) actually consists of millions of cells with varying size, residence time, vasculature and geometry. The resulting models which are nonlinear partial differential equations entail "axial-dispersion" type adipose tissue compartments to embody uncertain physiological heterogeneities in a single organism (rat). This models intra-individual variability due to the disperse nature of 
the micro structure in an individual. Most data (often even in vitro data) available for use in model fitting, however, involve aggregate data from a collection of individuals. This inter-individual variability is treated by viewing parameters (including intra-individual dispersion parameters) as random variables. One then attempts to estimate the associated distributions from aggregate data (multiple rat data) which also contains uncertainty (noise).

\subsection{Thermally Conductive Composite Adhesives}

Design methodology for composite adhesives is investigated in Bihari ${ }^{19}$, Banks and Bihari ${ }^{7}$ where the question of interest focuses on how to produce an adhesive with enhanced thermal conductivity. The objects of interest are epoxies and gels (with low thermal conductivity) filled with (highly conductive) particles, such as diamond dust, carbon, and aluminum. To pursue such studies, one employs models for heat transport (effective conductivity) in heterogeneous materials. Data from multiple samples are used to estimate parameters related to thermal properties of the composite materials. Also of importance in design of such materials are sensitivity analyses with respect to particle conductivity, geometry, etc. along with homogenization techniques. Both intraand inter- individual variability (due to varying particle sizes and non-uniform material samples) must be treated in these studies. This again results in parameters treated as random variables. Another aspect of uncertainty plays a role here since one is interested in properties of materials with randomly generated physical location of particles (i.e., uncertainty in location of particles) with distributions of particle size (uncertainty in particle size).

\subsection{Electromagnetic Imaging of Dielectric Materials}

The monograph Banks et $\mathrm{al}^{9}$ focuses on inverse problems involving electromagnetic detection. It initiates the development of computational techniques for pulsed microwave interrogation of targets to determine dielectric properties and geometry. Motivating applications include remote interrogation of military targets and non invasive medical diagnostics. Subsequent efforts (Banks and Raye ${ }^{15}$, Banks and Raye ${ }^{16}$, Banks and Raye ${ }^{17}$ ) involve the use of acoustic interfaces as reflecting devices in this developing technology. However, in practice the targets are usually heterogeneous, complex materials that are to be characterized by their polarization and conductivity properties. Therefore, in this class of problems, intra-individual variability arises due to multiple mechanisms at the basis of the polarization and conductivity. This in turn leads to a class of interesting inverse problems that can be considered in the 
generic framework that is the focus of our lecture here. Specifically, the model equations (Maxwell) are of the form (see Banks et $\mathrm{al}^{9}$ )

$$
\varepsilon_{r} \frac{\partial^{2} E}{\partial t^{2}}+\frac{1}{\varepsilon_{0}} \frac{\partial^{2} \mathcal{P}}{\partial t^{2}}+\frac{\sigma}{\varepsilon_{0}} \frac{\partial E}{\partial t}-c^{2} \frac{\partial^{2} E}{\partial z^{2}}=J
$$

with polarization $\mathcal{P}$ given in very general form by

$$
\mathcal{P}(t, z)=\int_{0}^{t} g(t-s, z) E(s, z) d z .
$$

For a heterogeneous material, the polarization $\mathcal{P}$ involves a "mixture" of mechanisms, represented by models such as the Debye model:

$$
\frac{d P_{1}}{d t}+\frac{1}{\tau} P_{1}=\frac{\varepsilon_{0}}{\tau}\left(\varepsilon_{s}-\varepsilon_{\infty}\right) E
$$

the Lorentz model:

$$
\frac{d^{2} P_{2}}{d t^{2}}+\frac{1}{\tau} \frac{d P_{2}}{d t}+\omega_{0}^{2} P_{2}=\varepsilon_{0} \omega_{p}^{2} E
$$

as well as higher order models and mechanisms. Hence the polarization $\mathcal{P}$ is made up of a distribution of the $P_{i}$ 's from such a family of differential equation systems. Thus, one seeks to estimate a random variable $\pi$ defined on a family of differential equation side constraints to the Maxwell system (i.e., the sample space is a family of differential equations!).

\subsection{Modeling of the HIV Infection Pathway}

The final example we present will also be used in the next section to outline and explain the application of a theoretical framework for the generic inverse problem introduced in Section 1. The HIV infection pathway, as depicted in Figure 1, is quite complex and entails a number of steps:

i. viral entry into target (uninfected cell);

ii. reverse transcription of viral RNA into DNA;

iii. transport of newly made DNA into the nucleus;

$i v$. integration of viral DNA into chromosome;

$v$. production of viral RNA and protein; and 
$v i$. creation of new virus from newly synthesized RNA molecules and proteins.

An important feature of numerous models (see Banks et $\mathrm{al}^{8}$, Bortz et $\mathrm{al}^{21}$ and the references therein) which appears to agree with these biological processes are the intracellular delays:

- $\tau_{1}$ : the time that a newly (acutely infected) cell takes to become a productively infected cell, and

- $\tau_{1}+\tau_{2}$ : the time that an acutely infected cell takes to become a chronically infected cell.

Typical model variables include $V$, the infectious viral population count, $A$ the number of acutely infected cells, $C$ the number of chronically infected cells, $T$ the number of uninfected target cells, and $X=A+C+T$, the total cell population count. Some models (especially those involving treatment and control) also entail immune response variables.

Models that account for intracellular delays usually involve systems of equations of the form (generally nonlinear)

$$
\frac{d V}{d t}=-c V(t)+n_{a} A(t-\tau)+n_{c} C(t)-n_{v t} V(t) T(t)
$$

where $\tau$ is a production delay which actually is distributed across the population of cells. That is, one should write

$$
\frac{d V}{d t}=-c V(t)+n_{a} \int_{0}^{\infty} A(t-\tau) k(\tau) d \tau+n_{c} C(t)-n_{v t} V(t) T(t)
$$

where $k$ is a probability density to be estimated from aggregate data.

Even if $k$ is given, these systems are nontrivial to simulate; this requires development of fundamental techniques Banks ${ }^{5}$, Banks and Kappel ${ }^{11}$ for both simulation and estimation. To be more precise, a specific model (see Banks et $\left.\mathrm{al}^{8}\right)$ is given by:

$$
\begin{aligned}
& \dot{V}(t)=-c V(t)+n_{A} \int_{0}^{r} A(t-\tau) d \pi_{1}(\tau)+n_{C} C(t)-p(V, T) \\
& \dot{A}(t)=\left(r_{v}-\delta_{A}-\delta X(t)\right) A(t)-\gamma \int_{0}^{r} A(t-\tau) d \pi_{2}(\tau)+p(V, T) \\
& \dot{C}(t)=\left(r_{v}-\delta_{C}-\delta X(t)\right) C(t)+\gamma \int_{0}^{r} A(t-\tau) d \pi_{2}(\tau) \\
& \dot{T}(t)=\left(r_{u}-\delta_{u}-\delta X(t)\right) T(t)-p(V, T)+S
\end{aligned}
$$


where

$$
C(t)=\mathcal{E}_{2}\{C(t ; \tau)\}=\int_{0}^{r} C(t ; \tau) d \pi_{2}(\tau),
$$

$A$ is the number of acute cells, $V(t)=V_{A}(t)+V_{c}(t)$,

$$
V_{A}(t)=\mathcal{E}_{1}\left\{V_{A}(t ; \tau)\right\}=\int_{0}^{r} V_{A}(t ; \tau) d \pi_{1}(\tau)
$$

is the number of virions at time $t$ produced by acutely infected cells, and $V_{c}(t)$ is the number of virions at time $t$ that have been produced by chronically infected cells. Here $\pi_{1}$ is the probability distribution for the delay from acute infection to viral production, and $\pi_{2}$ is the probability distribution for the delay from acute infection to chronic infection, $T$ is the number of cells, $X$ is the total (infected + uninfected) number of cells.

In the next section we present a theoretical framework and indicate how it can be applied to treat this example.

\section{A Theoretical Framework}

In order to develop a theoretical basis for the generic inverse problem of Section 1, one must develop some topological notions for a measure space over which to minimize the cost functional (2). For this we rely on some basics of probability theory as summarized in Banks and Bihari ${ }^{6}$ (see also Billingsley ${ }^{20}$ ).

We define $\Pi=\Pi(\mathcal{T})=\left\{\pi=\left(\pi_{1}, \pi_{2}\right): \pi_{i}\right.$ are probability measures on $\mathcal{T}=[0, r]\}$. Then $(\Pi(\mathcal{T}), \rho)$ is a metric space with Prohorov metric $\rho$. It is a complete metric space and is compact since $\mathcal{T}$ is compact. The Prohorov metric is not intuitively defined, but convergence in this metric can be stated in several simple and readily usable forms. They are equivalent and are given by:

i. $\rho\left(\pi^{k}, \pi\right) \rightarrow 0$

ii. $\int_{\mathcal{T}} g d \pi^{k} \rightarrow \int_{\mathcal{T}} g d \pi$ for all $g \in C(\mathcal{T})$;

iii. $\pi^{k}[A] \rightarrow \pi[A]$ for all Borel $A \subset \mathcal{T}$ with $\pi[\partial A]=0$.

For details on Prohorov metric and an approximation theory, see Banks and Bihari $^{6}$, Billingsley ${ }^{20}$. 
Once one has a topology on the space of random variables used as parameters, one can readily develop a general theoretical framework that includes the HIV models of Section 2.4. We consider a parameter $(\pi)$ dependent functional differential equation (FDE) system:

$$
\begin{aligned}
\frac{d x}{d t} & =f\left(t, x_{t}, \pi\right), \quad \pi \in \Pi, \\
x_{0} & =\phi \quad \text { where } \quad x_{t}(\theta)=x(t-\theta), 0 \leq \theta \leq r .
\end{aligned}
$$

One then assumes (or in the case of the HIV system (3) of Section 2.4, argues) that $(t, \psi, \pi) \rightarrow f(t, \psi, \pi)$ is

a. continuous from $[0, T] \times C[0, r] \times \Pi$ to $R^{n}$, and

b. locally Lipschitz in $\psi$.

Then by continuous dependence on "parameters" results for FDE's (an extension of standard ordinary differential equation results to FDE's with general vector space parameters, see Banks ${ }^{2}$, Banks ${ }^{3}$ for the basic ideas), one obtains that $\pi \rightarrow x(t ; \pi)$ is continuous from $\Pi$ to $R^{n}$ for each $t$. This yields

$$
\pi \rightarrow J(\pi, \hat{d})=\sum_{i}\left|C x\left(t_{i} ; \pi\right)-d_{i}\right|^{2}
$$

is continuous from $\Pi$ to $R^{1}$, where $\Pi=(\Pi(\mathcal{T}), \rho)$, is compact and $\rho$ is the Prohorov metric.

Then the general theory of Banks and Bihari ${ }^{6}$ can be followed to obtain existence and stability for inverse problems (continuous dependence with respect to data of solutions of the inverse problem). Moreover, an approximation theory is obtained that can be used as a basis for computational methods.

We can also obtain results for "method stability under approximation" (see Banks ${ }^{4}$, Banks and Kunisch ${ }^{12}$, Banks et $\mathrm{al}^{18}$ ). To briefly summarize these, let $\mathcal{T}_{M}=\left\{\tau_{j}^{M}\right\} \subset \mathcal{T}$ be such that $\cup_{M} \mathcal{T}_{M}$ is dense in $\mathcal{T}$ and define

$\Pi^{M}(\mathcal{T})=\left\{\pi_{M} \in \Pi: \pi_{M}=\sum_{j=1}^{M} p_{j} \delta_{\tau_{j}^{M}}, \tau_{j}^{M} \in \mathcal{T}_{M}, p_{j} \in R, p_{j} \geq 0, \sum p_{j}=1\right\}$.

Let $\hat{d}=\left\{d_{i}\right\}, \quad \hat{d}^{k}=\left\{d_{i}^{k}\right\}$ be sets of data (observations) such that $\hat{d}^{k} \rightarrow \hat{d}$ as $k \rightarrow \infty$. Define

$$
\Pi_{M}^{*}\left(\hat{d}^{k}\right)=\left\{\text { set of minimizers for } \quad J^{k}(\pi)=J\left(\pi, \hat{d}^{k}\right) \quad \text { over } \quad \Pi^{M}(\mathcal{T})\right\}
$$


and

$$
\Pi^{*}(\hat{d})=\{\text { set of minimizers for } \quad J(\pi, \hat{d}) \quad \text { over } \Pi(\mathcal{T})\} .
$$

Let $\operatorname{dist}(A, B)$ be the Hausdorff distance between sets $A$ and $B$. Then we have

Theorem 1 We have dist $\left(\Pi_{M}^{*}\left(\hat{d}^{k}\right), \Pi^{*}(\hat{d})\right) \rightarrow 0$ as $M \rightarrow \infty, \hat{d}^{k} \rightarrow \hat{d}$, so that solutions depend continuously on data and approximate problems are "method stable".

The above theory can be applied to the HIV model (3) given in Section 2.4. To see this, let $x=(V, A, C, T)$ and observe that the $\pi$ dependent terms in right sides of the system have the form $\int_{0}^{r} x(t-\tau) d \pi(\tau)$, so that continuity with respect to $\pi$ in the Prohorov metric is readily established, i.e., by the equivalence of $(i$.$) and (ii.) above, we have immediately$

$$
\int_{0}^{r} x(t-\tau) d \pi^{k}(\tau) \rightarrow \int_{0}^{r} x(t-\tau) d \pi(\tau) \quad \text { whenever } \quad \rho\left(\pi^{k}, \pi\right) \rightarrow 0 .
$$

The data is such that the $d_{i}$ are observations for $A\left(t_{i}\right)+C\left(t_{i}\right)+T\left(t_{i}\right)$, so that the remainder of the conditions needed for the theory to be applicable are readily satisfied.

In Banks et $\mathrm{al}^{8}$, we used experimental data to estimate the delays in the HIV models of Section 2.4 in the context of such a framework as described here. In these inverse problem calculations in Banks et $\mathrm{al}^{8}$ we used numerical approximation methods for the FDE's (both discrete delays and continuous probability density functions were used). The approximation methods were spline-based as developed in Banks ${ }^{5}$ and Banks and Kappel ${ }^{11}$.

In the results reported in Banks et $\mathrm{al}^{8}$, we estimated $p$ of the nonlinear term $p(V, T)$, and Dirac measures $\pi_{1}=\delta_{\tau_{1}}$ and $\pi_{2}=\delta_{\tau_{1}+\tau_{2}}$ as well as continuous probability density functions associated with the delays from acute infection to viral production and from acute infection to chronic infection. Extremely good fits to the experimental data were obtained.

\section{Concluding Remarks}

We close with several summary comments.

1. Randomness (uncertainty) is ubiquitous in inverse and estimation problems, whether due to uncertainty in modeling, intra- and inter-individual variability in aggregate data for populations, etc. or combinations of these. 
2. Important applications include: biology (PBPK models, HIV cellular infection models); materials (design of modern composites); electromagnetic interrogation (medical diagnostics, remote detection) as well as numerous others.

3. Successful efforts require combining deterministic and probabilistic modeling, and theoretical and computational ideas (mixing distributions, random effects).

4. Both theoretical and computational challenges are significant!!

Some initial efforts have been made, but much is yet to be done - see the program on Inverse Problem Methodology in Complex Stochastic Mod-

els (www.samsi.info) for Fall, 2002 at the new institute, the Statistical and Applied Mathematical Sciences Institute (SAMSI).

\section{Acknowledgements}

This research was supported in part by the U.S. Air Force Office of Scientific Research under grant AFOSR-F49620-1-00-0026.

\section{References}

1. R.A. Albanese, H.T. Banks, M.V. Evans and L.K. Potter, "PBPK models for the transport of trichloroethylene in adipose tissue", CRSC-TR01-03, Jan., 2001; Bull. Math Biology, to appear.

2. H.T. Banks, "Necessary conditions for control problems with variable time lags", SIAM J. Control, $\underline{6}$ (1968), 9-47.

3. H.T. Banks, "Variational problems involving functional differential equations", SIAM J. Control, $\underline{7}$ (1969), 1-17.

4. H.T. Banks, "On a variational approach to some parameter estimation problems", LCDS Technical Report \#85-14, May 1985; in Distributed Parameter Systems (F. Kappel, et. al.eds.) Springer Lecture Notes in Control and Info. Sci., $\underline{75}$ (1985), 1-23.

5. H.T. Banks, "Identification of nonlinear delay systems using spline methods", Proc. Intl. Conf. on Nonlinear Phenomena in Math. Sciences (V. Lakshmikantham, Ed.), Academic Press (1982), 47-55.

6. H.T. Banks and K.L. Bihari, "Modeling and estimating uncertainty in parameter estimation", CRSC-TR99-40, NCSU, Dec., 1999; Inverse Problems, 17 (2001), 1-17. 
7. H.T. Banks and K.L. Bihari, "Analysis of thermal conductivity in composite adhesives", CRSC-TR01-20, NCSU, August, 2001; Numerical Functional Analysis and Optimization, submitted.

8. H.T. Banks, D.M. Bortz and S.E. Holte, "Incorporation of variability into the modeling of viral delays in HIV infection dynamics", CRSC-TR01-25, Sept., 2001; Math Biosciences, submitted.

9. H.T. Banks, M.W. Buksas and T. Lin, Electromagnetic Material Interrogation Using Conductive Interfaces and Acoustic Wavefronts, SIAM Frontiers in Applied Mathematics, Vol. FR21, Philadelphia, 2000.

10. H.T. Banks, M.L. Joyner, B. Wincheski and W.P. Winfree, "Nondestructive evaluation using a reduced-order computational methodology", ICASE Tech Rep. 2000-10, NASA Langley Res. Ctr., March 2000; Inverse Problems 16 (2000), pp. 929-945.

11. H.T. Banks and F. Kappel, "Spline approximations for functional differential equations", J. Differential Equations, 34 (1979), 496-522.

12. H.T. Banks and K. Kunisch, Estimation Techniques for Distributed Parameter Systems, Birkhaüser Boston, 1989.

13. H.T. Banks and L.K. Potter, "Well-posedness results for a class of toxicokinetic models", CRSC-TR01-18, July, 2001; Discrete and Continuous Dynamical Systems, submitted.

14. H.T. Banks and L.K. Potter, "Model predictions and comparisons for three toxicokinetic models for the systemic transport of TCE", CRSCTR01-23, August, 2001; Mathematical and Computer Modeling, to appear.

15. H.T. Banks and J.K. Raye, "Computational methods for nonsmooth acoustic systems", CRSC-TR01-02, NCSU, January, 2001; Computational and Applied Mathematics to appear.

16. H.T. Banks and J.K. Raye, "Computational methods for nonsmooth acoustic systems arisin gin an electromagnetic hysteresis identification problem", Proceedings of the $18^{\text {th }}$ ASME Biennial Conference on Mechanical Vibration and Noise, Pittsburgh, PA, Sept. 9-12, 2001, to appear.

17. H.T. Banks and J.K. Raye, "Well-posedness for systems representing electromagnetic/acoustic wavefront interaction", CRSC-TR01-34, December, 2001; ESAIM: Control, Optimization and Calculus of Variations, submitted.

18. H.T. Banks, R.C. Smith and Y. Wang, Smart Material Structures: Modeling, Estimation and Control, 1996; Masson/John Wiley, Paris/Chichester.

19. K.L. Bihari, Analysis of Thermal Conductivity in Composite Adhesives, 
Ph.D. Thesis, NCSU, August, 2001.

20. B. Billingsley, Convergence of Probability Measures, Wiley, New York, 1968.

21. D. Bortz, R. Guy, J. Hood, K. Kirkpatrick, V. Nguyen and V. Shimanovich, "Modeling HIV infection dynamics using delay equations", in $6^{\text {th }}$ CRSC Industrial Math Modeling Workshop for Graduate Students, NCSU (July, 2000), CRSC-TR00-24, Oct., 2000.

22. L.K. Potter, Physiologically Based Pharmacokinetic Models for the Systemic Transport of Trichloroethylene, Ph.D. Thesis, NCSU, August, 2001. 


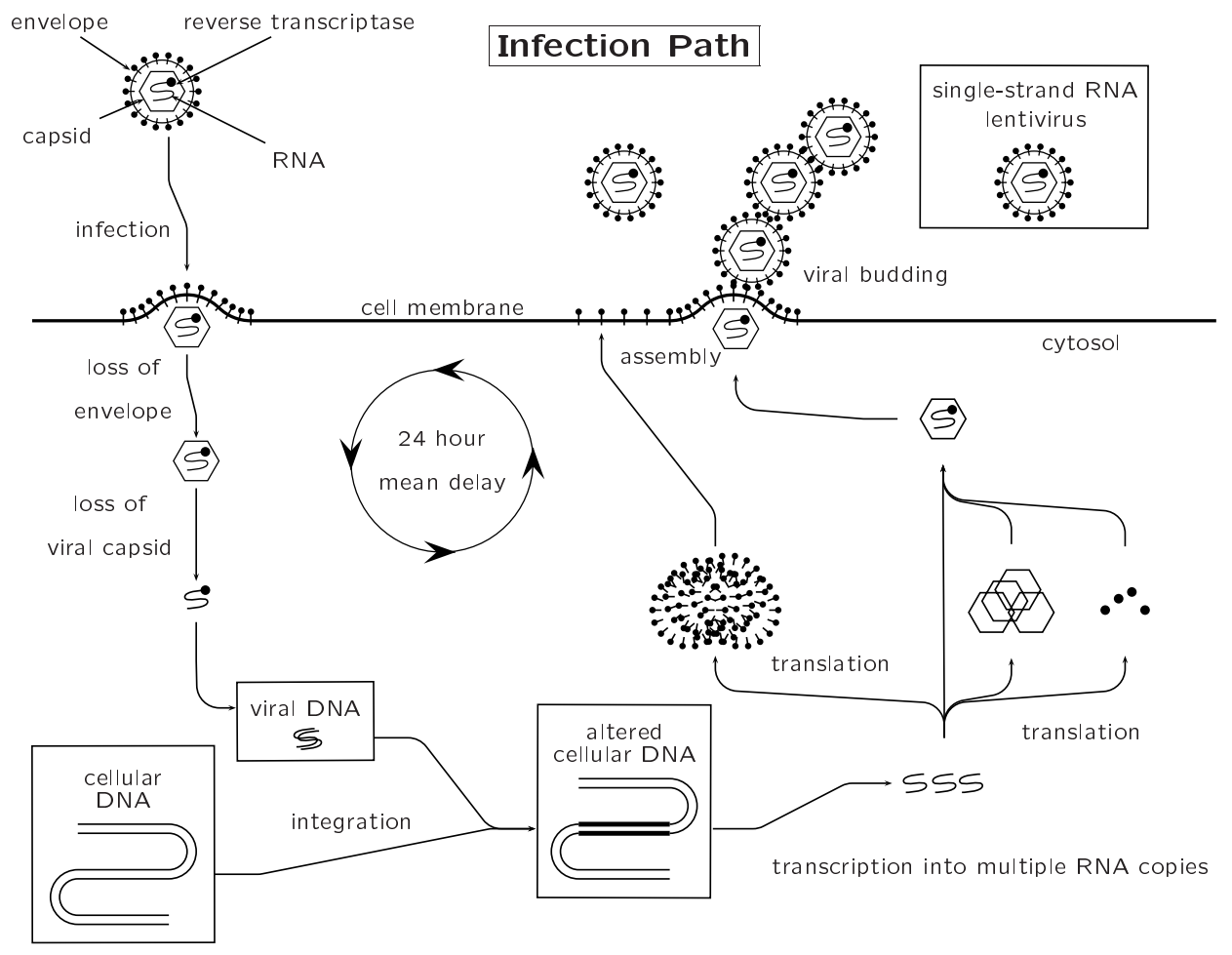

Figure 1. HIV Infection Pathway 